\title{
Design, Simulation, and Performance Analysis of an INS/GPS System using Parallel Kalman Filters Structure
}

\author{
Tran Duc-Tan ${ }^{1}$, Paul Fortier ${ }^{2}$, Huu-Tue Huynh ${ }^{3}$ \\ ${ }^{1}$ University of Engineering and Technology, Vietnam National University, Hanoi, Vietnam \\ ${ }^{2}$ Laval University, Canada \\ ${ }^{3}$ Bac Ha International University, Vietnam \\ Correspondence: Tran Duc-Tan, tantd@vnu.edu.vn \\ Manuscript communication: received 11 April 2010, revised 15 June 2011, accepted 28 June 2011
}

\begin{abstract}
Thanks to the strong growth of MEMS technology, the Inertial Navigation System (INS) is widely applied to navigation and guidance of moving objects. However, there exist errors in the inertial sensor's signals that cause unacceptable drifts. To minimize these effects on the INS system, a GPS is usually employed simultaneously with an INS in order to increase the dimension of the system; the desired parameters are estimated by Kalman filtering technique applied to the enlarged system. In this paper, we present the design, simulation and performance analysis of an INS/GPS system using two parallel Kaman filters in order to increase the accuracy of the parameter estimation process. The results show that this system could be efficiently brought to practical applications.
\end{abstract}

Keywords- GPS/INS integration, Kalman filtering, parallel Kalman filters structure, low-cost navigation systems.

\section{INTRODUCTION}

Nowadays, navigation and control of automobiles, ships and aerial vehicles are important and widely used in civil and military applications [1, 2]. One of the mature technologies for navigation is the global positioning system (GPS) with the advantages of high accuracy and time independence positioning. However, GPS navigation is suffering from signal losses due to geographic obstacles as well as selective availability (SA) in special cases. On the other hand, Inertial Navigation System (INS) uses inertial sensors such as accelerometers and gyroscopes to measure and calculate navigation information. With the development of Micro Electronic and Mechanical Systems (MEMS), these sensors can be produced at low cost, with small dimensions, low power consumption and fast response time allowing continuous update of navigation parameters [2]. Nevertheless, MEMS based INS systems exhibit several problems such as time dependent drift, instability with temperature, etc. To solve these problems, INS and GPS systems can be integrated into one global system to take advantages of both systems and can be widely applied in many fields such as unmanned vehicles for environmental monitor or surveillance and rescue. With this aim, our research group has developed models for INS/GPS integrated systems using MEMS sensors and Kalman filters to compensate the errors of MEMS sensors and to predict the navigation trajectory when the GPS signal is lost due to outage [3].

In Section 2, we review achievements obtained by researchers in integrating INS/GPS, especially for low cost systems. Section 3 presents the INS and GPS systems as well as our proposed scheme. Simulation results are presented in Section 4 . Section 5 and 6 show experimental results in the laboratory and outdoor environments, respectively. Section 7 concludes the paper with a general discussion on the results and remarks for future works.

\section{Literature Survey}

The heart of the integration of GPS and INS is the Kalman filter (KF) that can offer a combined system with higher performance in comparison to either system in stand-alone mode. A Kalman filter is a linear estimator that uses information of the current system's dynamics and measured data to obtain an optimal estimate of the state variables [4].

The integration schemes can be divided into two classes: loosely coupled and tightly coupled strategies [5]. In a tightly coupled scheme, all measured data from GPS and INS are processed in one filter. This kind of scheme provides the advantage of preserving data availability and removing poor GPS measurements. However, this scheme is more complex than the loosely coupled scheme [6].

Developing navigation systems for land navigation has attracted many researchers [7, 8]. In [9], the performance of the integration system in challenging GPS environments is given. Furthermore, there is a need to introduce integration systems with low price; the use of MEMS based IMU is a good solution due to their low cost. Shin et al. have used a velocity-matching alignment as the calibration method to bridge the GPS gap [10]. Mohamed et al. have developed an adaptive 
algorithm to estimate the system's noise matrix and the measurement noise covariance matrix for INS error estimation [11]. Recently, Fournier et al. have proposed an adaptive algorithm using wavelet for INS error estimation [12]. However, all these algorithms have to face the problem of real time requirements. The aim of our work is to develop a real time low-cost navigation system using aparallel Kalman filters structure in order to apply INS/GPS in real situations.

\section{Proposed Scheme}

\subsection{Inertial Navigation System (INS)}

An INS system often consists of three accelerometers and three gyroscopes in order to measure the accelerations in three dimensions and the rotation rates around three axes. The development of MEMS technology has been a stimulus to widen the application area of INS. Today, an Inertial Measurement Unit (IMU) even includes a three-degree of freedom gyroscope and a three-degree of freedom accelerometer [13, 14].

There are two typical INS systems: gimble and strapdown [15]. The strapdown INS system is more popular than the gimble system and is mostly based on MEMS technology that is relatively inexpensive and compact. In the strapdown system, accelerometers and gyroscopes are fixed to the frame of the aircraft. Signals from these sensors are processed in order to obtain three Euler angles. The results are corrected by gravity acceleration and Earth rotation velocity.

The orientation of the aircraft is determined by three Euler angles $(\psi, \theta, \phi)$, as illustrated on Figure 1. The following equation shows the relation between velocities of roll, pitch and yaw ( $p, q$ and $r)$, and the three Euler angles [15]:

$$
\left[\begin{array}{l}
\dot{\phi} \\
\dot{\theta} \\
\dot{\psi}
\end{array}\right]=\left(\begin{array}{ccc}
1 & \sin \phi \tan \theta & \cos \phi \tan \theta \\
0 & \cos \phi & -\sin \phi \\
0 & \sin \phi \sec \theta & \cos \phi \sec \theta
\end{array}\right)\left[\begin{array}{l}
p \\
q \\
r
\end{array}\right]
$$

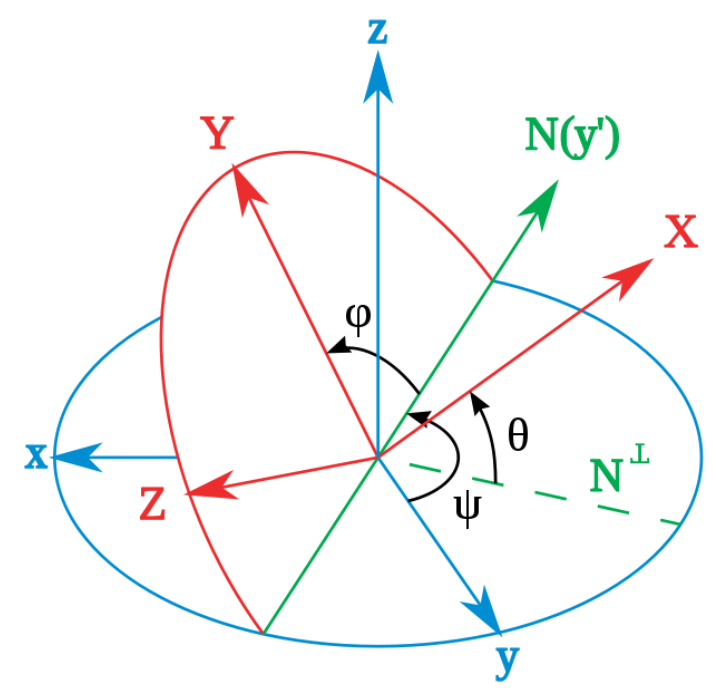

Figure 1. Three Euler angles [15].

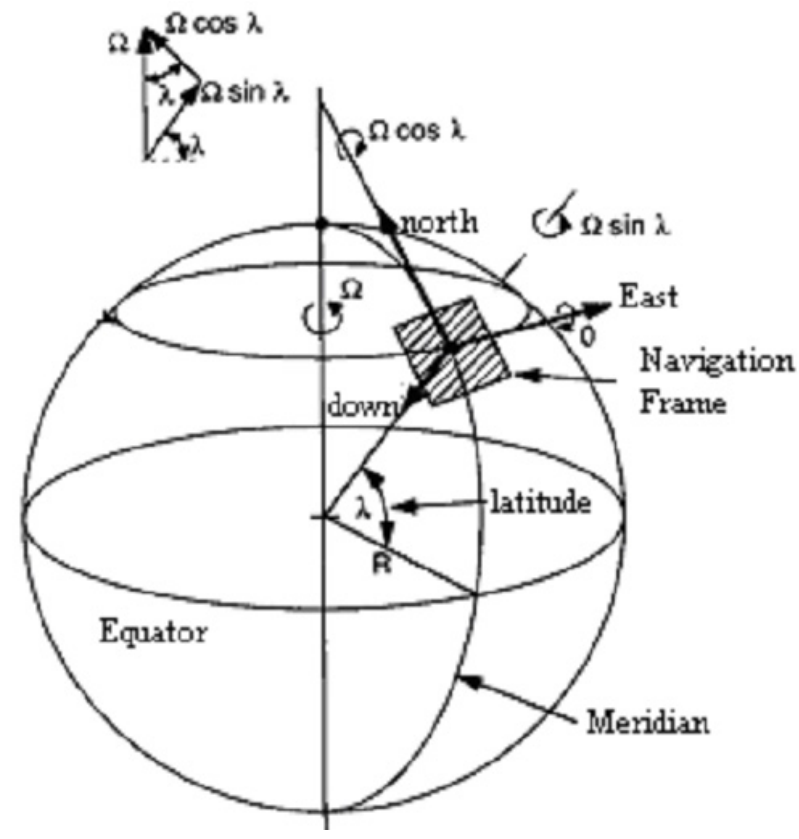

Figure 2. Navigation frame [15].

Table I

COMMON ERRORS IN THE INS SYSTEMS

\begin{tabular}{|l|l|}
\hline \multicolumn{1}{|c|}{ Type } & \multicolumn{1}{c|}{ Errors } \\
\hline Alignment & Roll, pitch, yaw \\
\hline Gyroscope & Bias, drift and scale factor errors \\
\hline Accelerometer & Bias, scale factor error \\
\hline Random noise & Random error \\
\hline
\end{tabular}

Equation (1) is then integrated to obtain the three Euler angles. Three accelerations $\left(a_{x}, a_{y}\right.$ and $\left.a_{z}\right)$ along the three axes of body frame relate to three velocities $(U, V$ and $W)$ in the Earth fixed frame by the following equations:

$$
\begin{aligned}
\dot{\mathbf{U}} & =a_{x}+V \cdot r-W \cdot q+g \cdot \sin \theta \\
\dot{\mathbf{V}} & =a_{y}-U \cdot r+W \cdot p-g \cdot \cos \theta \sin \varphi \\
\dot{\mathbf{W}} & =a_{z}-U \cdot q+W \cdot p-g \cdot \cos \theta \cos \varphi
\end{aligned}
$$

After integrating (2), we can obtain $U, V$ and $W$. Using the Direct Cosine Matrix (DCM), as given by Equation (3) at the beginning of the next page we can convert the movement from the Earth fixed frame to the navigation frame (see Figure 2) by:

$$
\left[\begin{array}{c}
\dot{X} \\
\dot{Y} \\
\dot{Z}
\end{array}\right]=\left[\begin{array}{c}
V_{N} \\
V_{E} \\
V_{D}
\end{array}\right]=D C M^{T}\left[\begin{array}{c}
U \\
V \\
W
\end{array}\right]
$$

We can position the aircraft by integrating (4). Then, we can obtain the latitude, longitude and height of the aircraft using the following:

$$
\dot{\lambda}=\frac{V_{N}}{R_{\text {earth }}} ; \quad \dot{\mu}=\frac{V_{E}}{R_{\text {earth }} \cdot \cos \lambda} ; \quad \dot{H}=-V_{D} ;
$$

There are many kinds of errors in INS systems. Most errors are caused by sensor imperfections. The most common errors in strapdown INS systems are summarized in Table I. A discrete INS algorithm was 


$$
D C M=\left[\begin{array}{ccc}
\cos \theta \cos \psi & \cos \theta \sin \psi & -\sin \theta \\
\sin \phi \sin \theta \cos \psi-\cos \phi \sin \psi & \sin \phi \sin \theta \sin \psi+\cos \phi \cos \psi & \sin \theta \cos \theta \\
\cos \phi \sin \theta \cos \psi+\sin \phi \sin \psi & \cos \phi \sin \theta \sin \psi-\sin \phi \cos \psi & \cos \theta \cos \theta
\end{array}\right]
$$

developed in our laboratory in order to develop a complete Inertial Measurement Unit (IMU). However, the details of this discrete algorithm will not be presented in this paper.

\subsection{Global Positioning System (GPS)}

The GPS system consists of 24 satellites, which fly above the Earth at a height of $19200 \mathrm{~km}$ in order to acquire the position of an aircraft (latitude, longitude and height) [4]. However, buildings, tunnels, etc. often block radio signals. To get the correct position of the aircraft requires at least four satellites. GPS can be separated in three segments (see Figure 3):

- Space segment: consisting of 24 satellites;

- Control segment: the ground control;

- User segment: the receiver (civil and military purposes).

Errors in GPS are mostly caused by the following six factors (not including selective availability error): ephemeris data, satellite clock, multipath reflection, atmospheric delay, random measurement noise and receiver (including software).

\subsection{Integration of INS and GPS using Kalman Filtering}

The INS system has two main advantages when compared with other navigation systems: self-contained ability and high accuracy for short-term navigation. However, a serious problem of INS is the accumulation of gyroscope and accelerometer errors. Therefore, in long-term navigation applications, INS works with the aid of other systems such as radio navigation systems

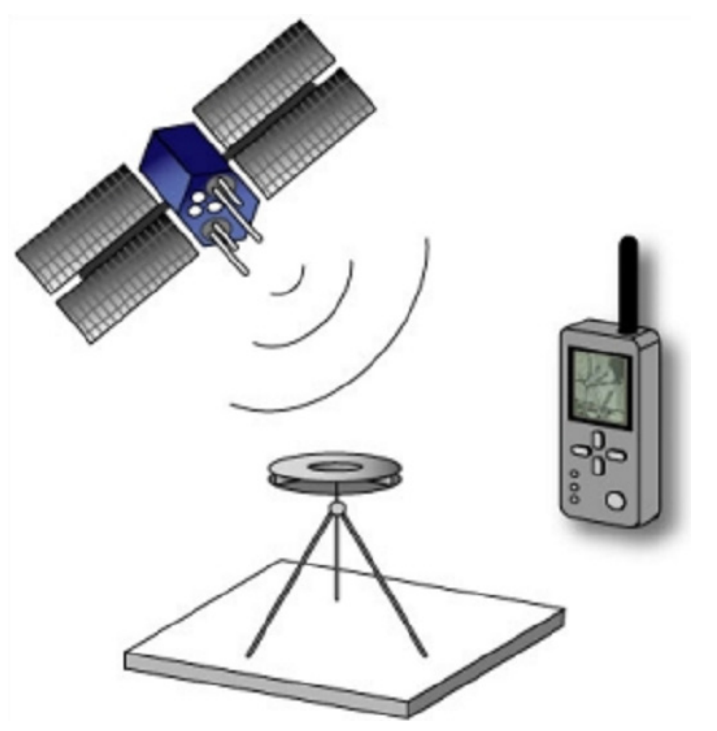

Figure 3. Structure of GPS [16].
(Loran, Tacan) or satellite navigation systems (GPS, GLONASS). The important advantage of these systems is that their performance is stable. Consequently, there is a great need for integration of INS and one of these systems. The integration of INS and GPS is considered as a good combination. The heart of the integrated system is the Kalman algorithm [17].

As mentioned before, the aim of this paper is to develop a specific scheme for INS/GPS integration that can be used in the case where the GPS signal gets most frequently lost. The integration system based on two parallel Kalman filters is developed and tested. The INS/GPS system can switch between feed-forward and feedback schemes depending on GPS environments. The INS error estimation scheme is shown in Figure 4. The INS error equations are used as a system model and the measured input data fed to the filter are the differences between the INS and GPS positions and velocities. When GPS data is not available, the Kalman filter works in prediction mode and the INS/GPS system switch to the feed-forward scheme.

Solving the navigation problem can be divided into two parts: creating a process model of the navigation system and modeling the IMU's sensors. The process model describes the prediction of states, which are the position, the velocity, the attitude, etc.

In discrete form, any linear system can be described as:

$$
x_{k}=A_{k, k-1} x_{k-1}+G_{k, k-1} w_{k-1},
$$

where $A_{k, k-1}$ is a transition matrix, $G_{k, k-1}$ is an $n \times r$ input matrix, and $w_{k}-1$ is an $r \times 1$ input noise. We can derive these matrices based on the INS error equations. The measurement model is given by:

$$
z_{k}=H_{k} x_{k}+v_{k}
$$

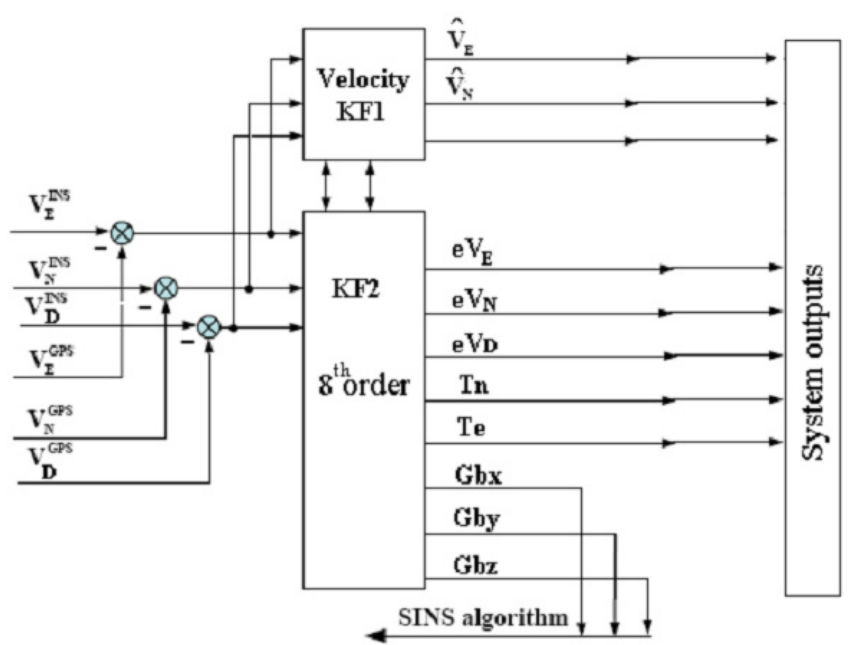

Figure 4. The integration configuration. 
where $z_{k}$ is an $m \times 1$ measurement vector, $H_{k}$ is an $m \times n$ design matrix, and $v_{k}$ is an $m \times 1$ measurement noise. All the measurement noises from GPS and IMU are assumed to be white Gaussian stationary process.

In block KF1, a conventional Kalman filter with a reduced system model is used for the INS velocity error estimation. Estimated INS velocity errors are compensated in the system output.

In block KF2, the estimation of INS errors is performed in order to improve estimation accuracy. There are eight such states $\left(x_{k}\right)$, which consist of attitude errors $\left(T_{n}, T_{e}\right)$, velocity errors $\left(e_{V N}, e_{V E}, e_{V D}\right)$, and drift terms $\left(G_{b x}, G_{b y}, G_{b z}\right)$. The INS errors are used to correct the elements of the transformation matrix $C_{b}^{N}$ and the quaternion. Estimated gyro drifts are also taken into account in the SINS navigation scheme. The transition matrix is:

$$
\begin{aligned}
& A_{k, k-1}= \\
& {\left[\begin{array}{cccccccc}
0 & 0 & 0 & 0 & 0 & h_{N} C_{11} & h_{N} C_{12} & h_{N} C_{13} \\
0 & 0 & 0 & 0 & 0 & h_{N} C_{21} & h_{N} C_{22} & h_{N} C_{23} \\
0 & -D_{v d} & 0 & 0 & 0 & 0 & 0 & 0 \\
D_{v d} & 0 & 0 & 0 & 0 & 0 & 0 & 0 \\
0 & 0 & 0 & 0 & 0 & 0 & 0 & 0 \\
0 & 0 & 0 & 0 & 0 & -h_{N} \beta & 0 & 0 \\
0 & 0 & 0 & 0 & 0 & 0 & -h_{N} \beta & 0 \\
0 & 0 & 0 & 0 & 0 & 0 & 0 & -h_{N} \beta
\end{array}\right],}
\end{aligned}
$$

where $D_{v d}$ is the velocity increment in the down direction of the navigation frame (north, east, down), $\beta$ is one of the parameters of the correlation function, and $h_{N}$ is $0.015625 \mathrm{~s}$.

In the case of GPS signal outage, the INS provides positioning until the GPS signal is reacquired. During such periods, navigation errors increase rapidly with time due to the time-dependent INS error behavior. The reason is that the low cost MEMS IMUs consists of low quality sensors that introduce large errors and noises. In these cases, we sometime use land vehicle motion attributes to prevent INS error accumulation. The information derived from the behavior of a land vehicle will compensate the GPS's measurements. In this paper, we consider velocity and height constraints. They can provide virtual measurements to aid the IMU.

We can only correct the attitude of the IMU using the attitude errors predicted by the state matrix. This corrected attitude forms a part of the whole system. To take the in-motion alignment of the navigation system, we use the heading from the GPS or the external heading measurements, such as from a magnetometer, into the attitude computations.

To ensure the quality of the whole system, the integration system was verified in three processed as shown in Figure 5.

\section{Simulations}

SIMULINK provides an efficient design and simulation tool for creating system level algorithm models that directly assist in implementation. The whole system has been simulated and tested by embedded programming

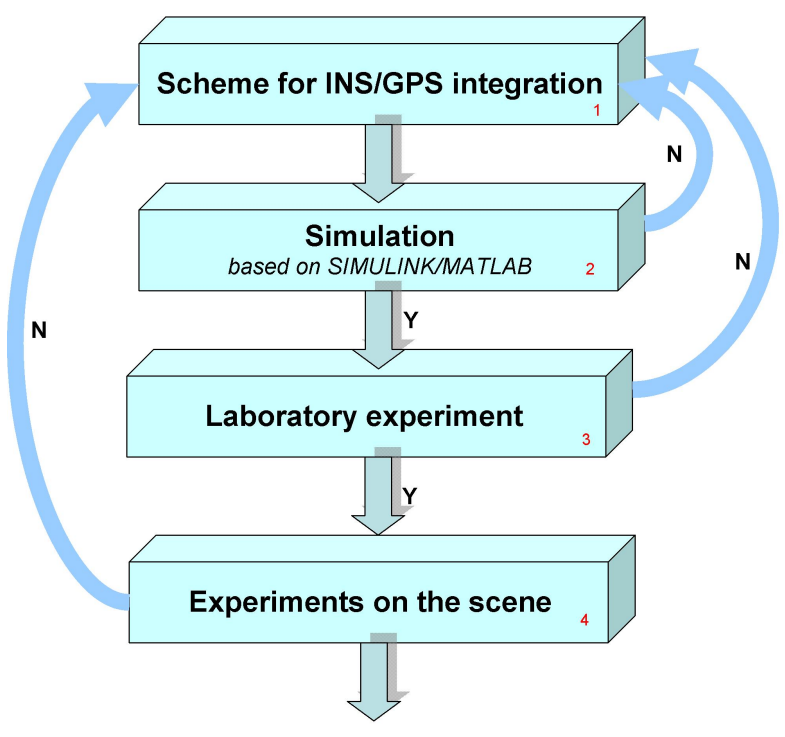

Figure 5. Flow chart of the INS/GPS implementation.

in the SIMULINK/MATLAB environment (see Figure 6). In this case, we have used the advantages of both SIMULINK and M-files. Thus, it is easy to develop a real-time hardware simulator. The simulation is conducted based on real data, which was measured from a vehicle equipped with a GPS and an IMU BP3010. A lot of experimental data were provided to demonstrate the behavior of the model at various times.

As mentioned above, a Kalman filter was used to estimate the INS errors. The INS can give out correct values of the position by subtracting from the noisy INS the estimation of the INS errors.

The reference trajectory mentioned in this paper was obtained using the Aerosim Blockset toolbox. Figure 7 shows the position of the aircraft along the north and east directions on the Earth instead of latitude and longitude. We can see that the unaided INS deviates from the ideal trajectory by a large distance. The Kalman filter can trace the ideal trajectory quite well.

\section{LABORATORY EXPERIMENT}

\subsection{Noise Characterization}

There are two kinds of noise in the INS: deterministic and stochastic. A careful calibration process usually eliminates the deterministic noise, but the stochastic noise is always difficult to treat. We have determined successfully the characteristics of the MEMS sensors' noise by analyzing the Allan variance of the experimental data. After characterizing the IMU errors, the information of these noises is applied to the KF based MEMS INS/GPS integration module in order to estimate the velocity, position and attitude of the aircraft. In this work, as for the INS system, we have used an IMU BP3010 [18], which consists of three ADXRS300 gyros and three ADXL210E accelerometers. The measurements are obtained by the IMU's microcontrollers and transmitted out via an RS232 interface. The unit transmits output data as angular incremental 


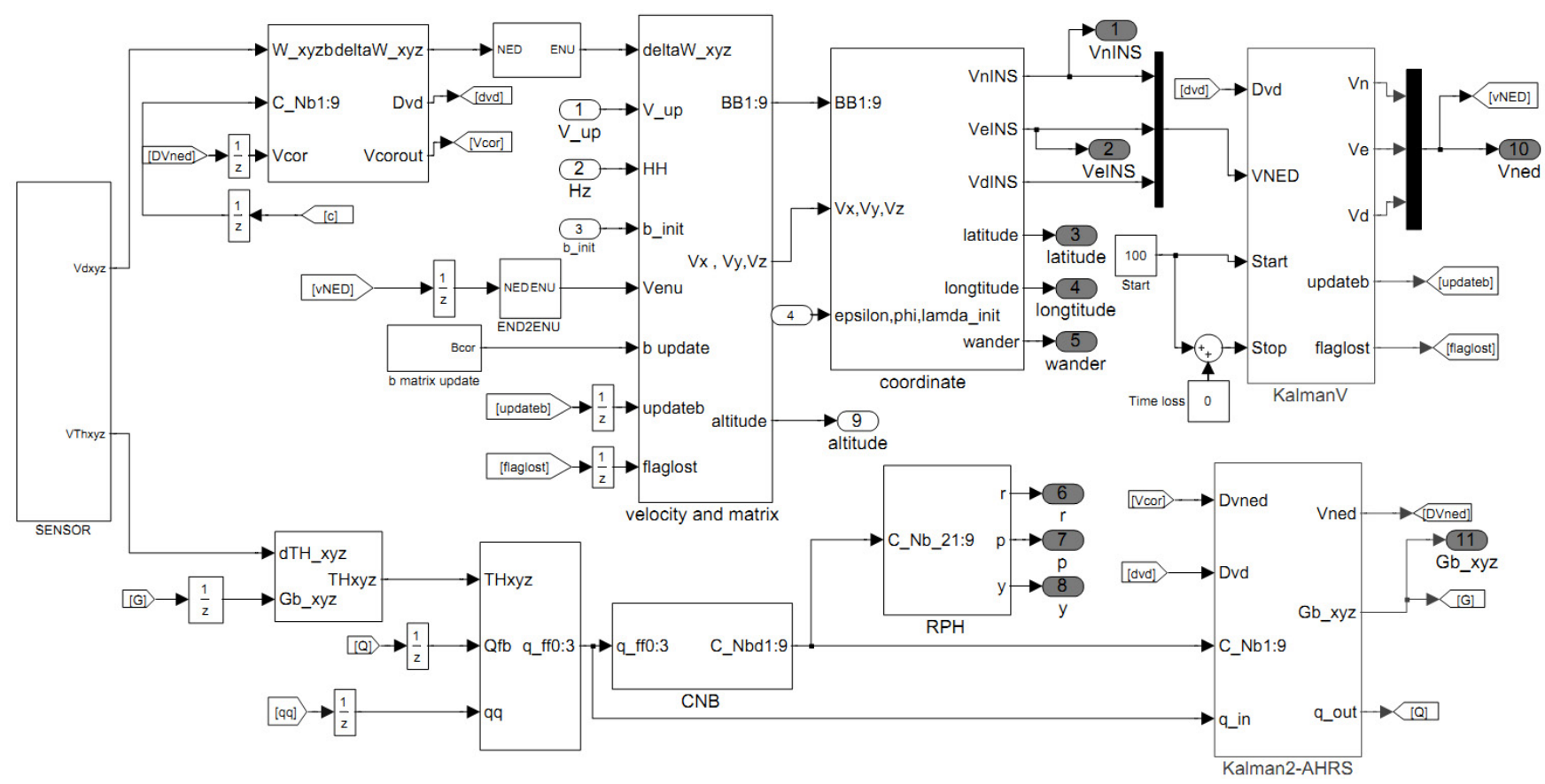

Figure 6. Flow chart of the INS/GPS implementation.

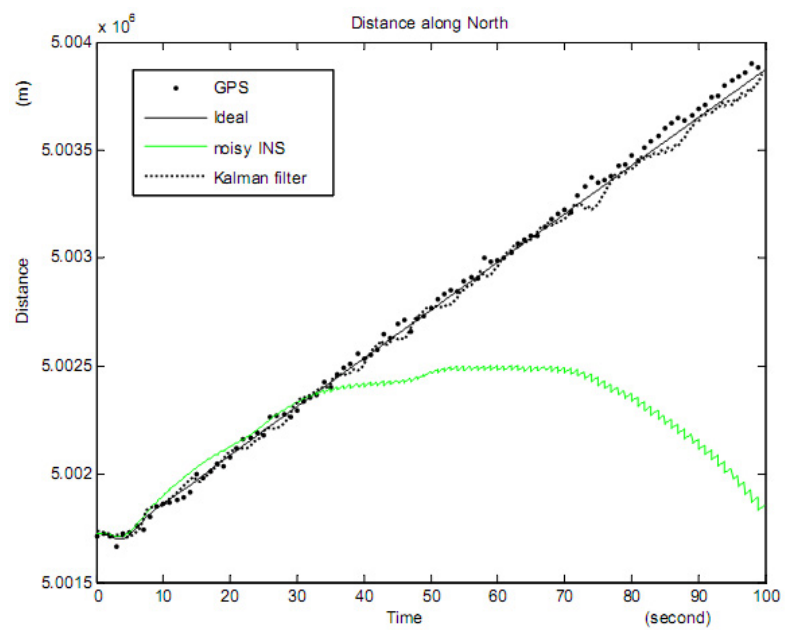

(a)

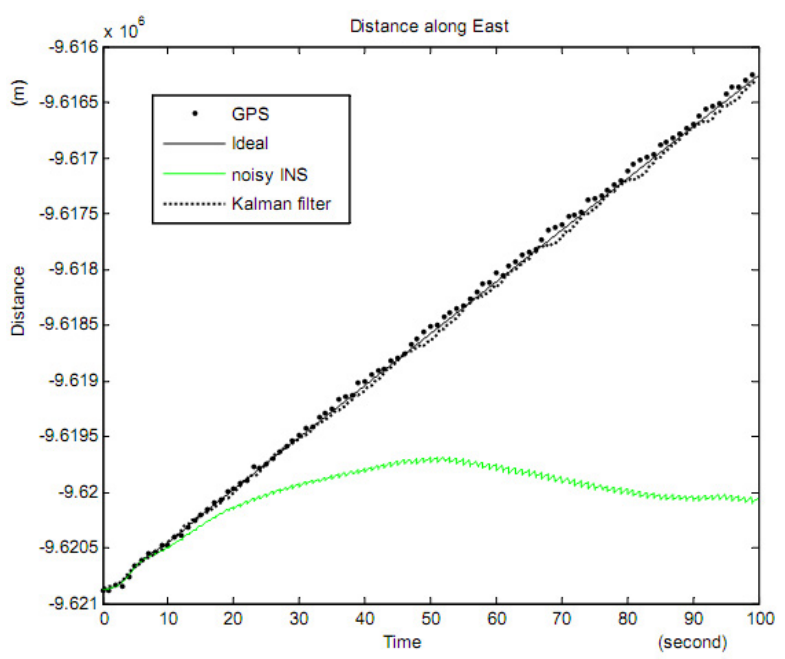

(b)

Figure 7. Distance along north (a) and east (b) and velocity incremental data in serial frames of 16 bytes at a frequency of $64 \mathrm{~Hz}$.

The position error of an INS increases rapidly with navigation due to the integration of measurement errors in the gyroscopes and accelerometers. In order to make the corrections, the errors are classified into deterministic errors and stochastic errors [19, 20].

To eliminate the deterministic errors, we can specify them quantitatively by calibrating the device. It is, however, more complex to estimate the stochastic errors. An optimal filter such as a Kalman filter is often used. In this case, the parameters of these stochastic errors must necessarily be specified. In this paper, we have determined the noise parameters of both deterministic and stochastic errors of MEMS based IMUs. For the deterministic errors, a precise rate table has been used as a calibration device. For the stochastic errors, we have used the Allan variance method. The Allan variance can reveal more information than the power spectrum density. Using the Allan variance method, we can obtain a reliable noise model that is applied directly to the Kalman filter. Table II shows the estimated noise coefficients for the gyros and the accelerometers. The $\times$ character means that the sensor lacks the error or this error is much smaller than the others. Furthermore, not only the intrinsic errors of the sensors but also the vibration caused by the vehicle's engine have been carefully analyzed in our scheme [21].

\subsection{Laboratory Experiments}

After a calibration procedure, the experiment system is placed on a precise rotation table, which performs a sequence of different rotation rates for each dimension. The IMU is initially positioned in the center of the rotation table and each rotation rate is run for approximately 10 minutes. 
Table II

Identified Noise Coefficients using Allan Variance

\begin{tabular}{|c|c|c|c|c|c|}
\hline Gyros & $\begin{array}{c}Q_{z}(\mathrm{rad}) \\
\text { (Quantization noise) }\end{array}$ & $\begin{array}{l}Q(\operatorname{rad} / \sqrt{s}) \\
\text { (White noise })\end{array}$ & $\begin{array}{c}B(\mathrm{rad} / \mathrm{s}) \\
\text { (Flicker noise) }\end{array}$ & $\begin{array}{l}K(\mathrm{rad} / \mathrm{s} / \sqrt{s}) \\
(\text { Random walk) }\end{array}$ & $\begin{array}{l}R\left(\mathrm{rad} / \mathrm{s}^{2}\right) \\
(\text { Trend })\end{array}$ \\
\hline$X$ & $1,504 * 10^{-6}$ & $1,368 * 10^{-5}$ & $x$ & $5,617 * 10^{-7}$ & $\times$ \\
\hline$Y$ & $1,655 * 10^{-6}$ & $1,517 * 10^{-5}$ & $5,315 * 10^{-6}$ & $x$ & $x$ \\
\hline Z & $1,668 * 10^{-6}$ & $1,535 * 10^{-5}$ & $5,556 * 10^{-6}$ & $4,892 * 10^{-7}$ & $\times$ \\
\hline Accelerometers & $Q_{z}(\mathrm{~m} / \mathrm{s})$ & $Q(\mathrm{~m} / \mathrm{s} / \sqrt{\mathrm{s}})$ & $B\left(\mathrm{~m} / \mathrm{s}^{2}\right)$ & $K\left(\mathrm{~m} / \mathrm{s}^{2} / \sqrt{\mathrm{s}}\right)$ & $R\left(\mathrm{~m} / \mathrm{s}^{3}\right)$ \\
\hline$x$ & $1,352 * 10^{-5}$ & $4,734 * 10^{-5}$ & $4,155 * 10^{-5}$ & $1,161 * 10^{-5}$ & $\times$ \\
\hline$y$ & $1,400 * 10^{-5}$ & $5,169 * 10^{-5}$ & $4,713 * 10^{-5}$ & $7,588 * 10^{-6}$ & $5.0685 * 10^{-7}$ \\
\hline$z$ & $1,339 * 10^{-5}$ & $5,688 * 10^{-5}$ & $4,025 * 10^{-5}$ & $9,197 * 10^{-6}$ & $7.4025 * 10^{-7}$ \\
\hline
\end{tabular}

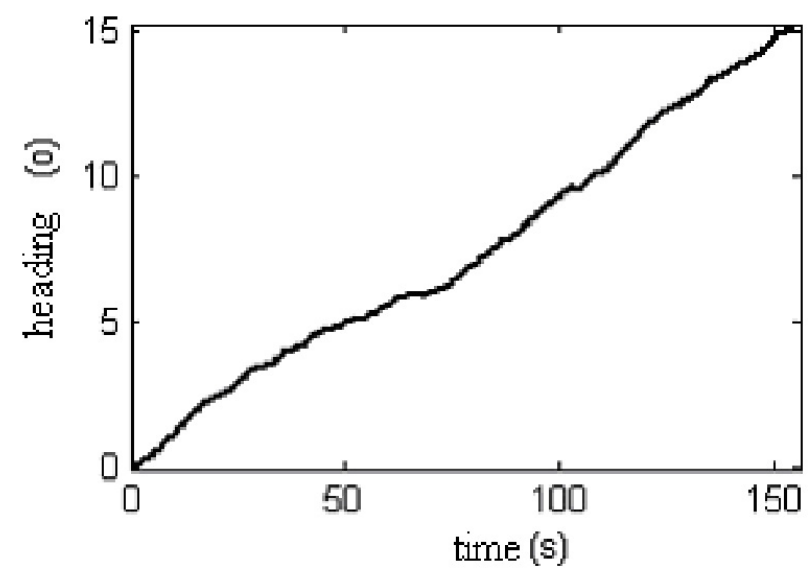

Figure 8. Heading showing drift (without KF).

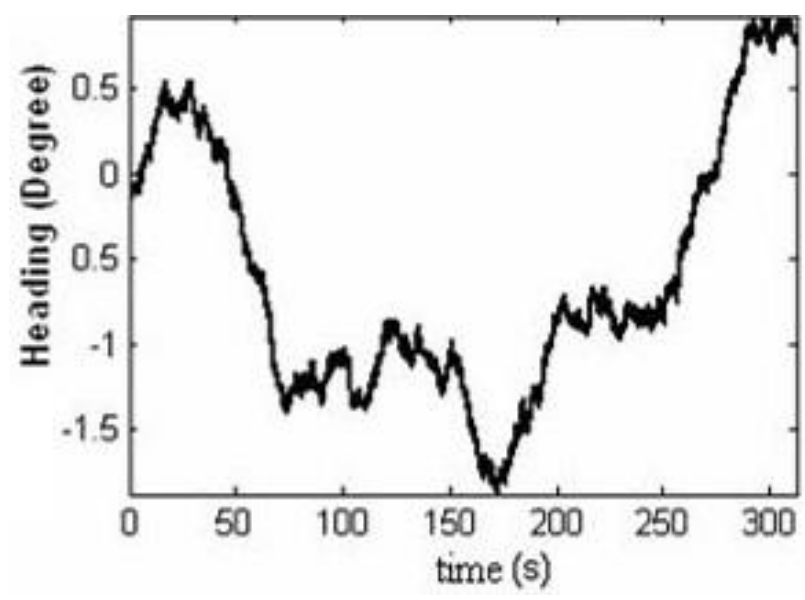

Figure 9. Heading in the presence of KF.

As mentioned above, eight states were considered and a Kalman filter was used to estimate the INS errors. The INS can give correct values of the velocities by subtracting from the noisy INS the estimation of the INS errors. To point out the usefulness of the KF, look at Figure 8 and Figure 9 that illustrate the heading of INS while standing still in the case without KF and with KF. While the INS is in rotation at a rate of $10^{\circ} / \mathrm{s}$, the integration system with KF can exactly provide the heading as shown in Figure 10.

Let us come back to the fixed case and look at the north velocities in two cases: with and without KF. The graphs for velocity computed and corrected by the Kalman filter are given in Figure 11. We can see that

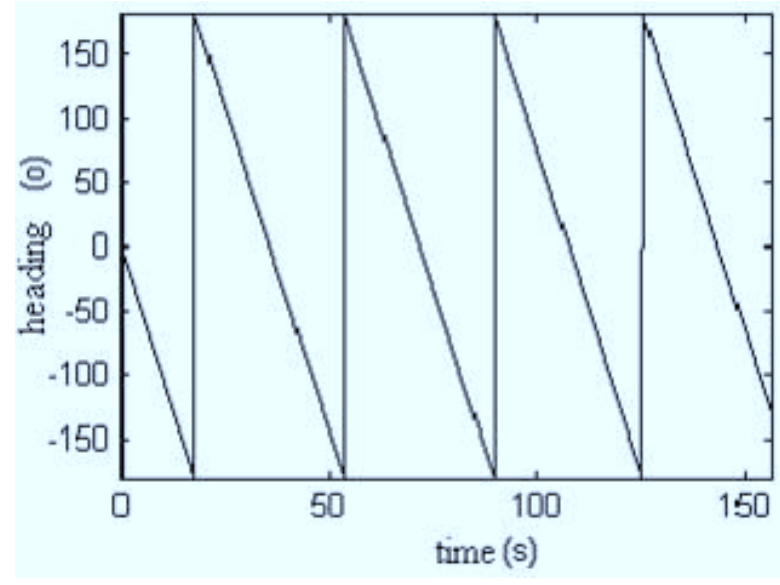

Figure 10. The heading with $\mathrm{KF}$ at a rotation rate of $10^{\circ} / \mathrm{s}$.

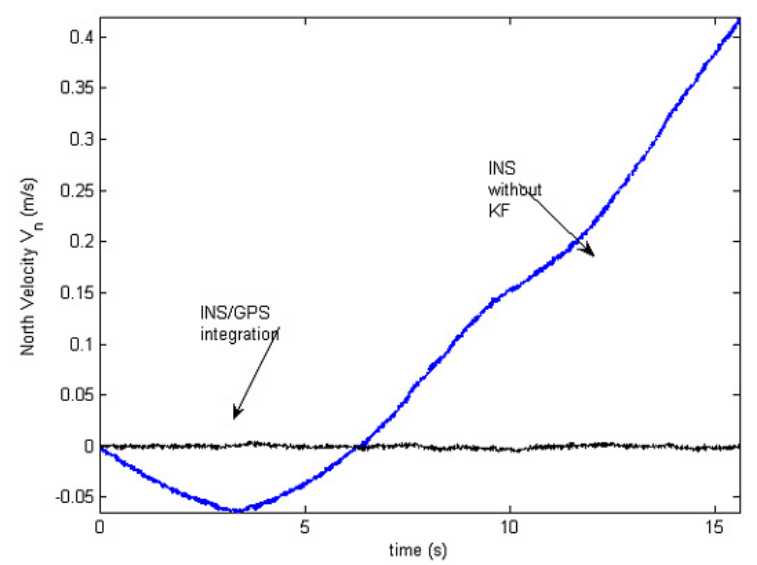

Figure 11. The north velocity of the fixed IMU in two cases: with and without KF.

the unaided INS deviates from the ideal velocity by a large quantity. If the integration system is supported by $\mathrm{KF}$, the output $\mathrm{Vn}$ is around $0 \mathrm{~m} / \mathrm{s}$. This means that the KF can provide the exact correction in the feed-forward configuration.

\section{Experiments using a Moving Vehicle}

In this section, a KF will be used for the INS/GPS integration system, which is mounted in a car. The land vehicle kinematics data set is also used during induced GPS outages. By using these methods, the results showed remarkable improvement of position errors. 


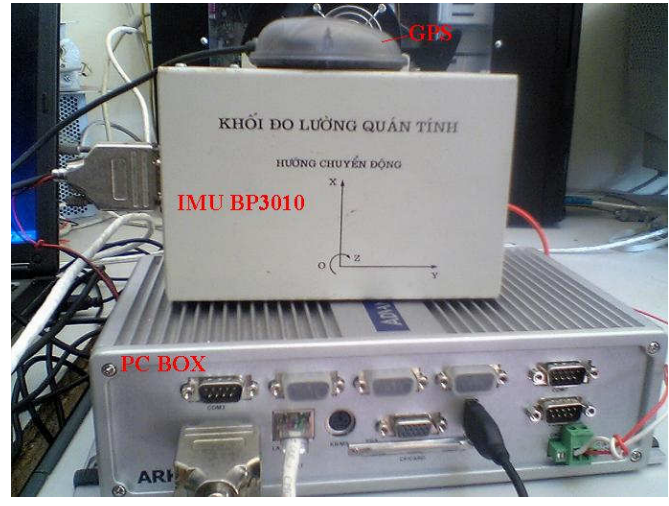

Figure 12. Hardware for the proposed system.

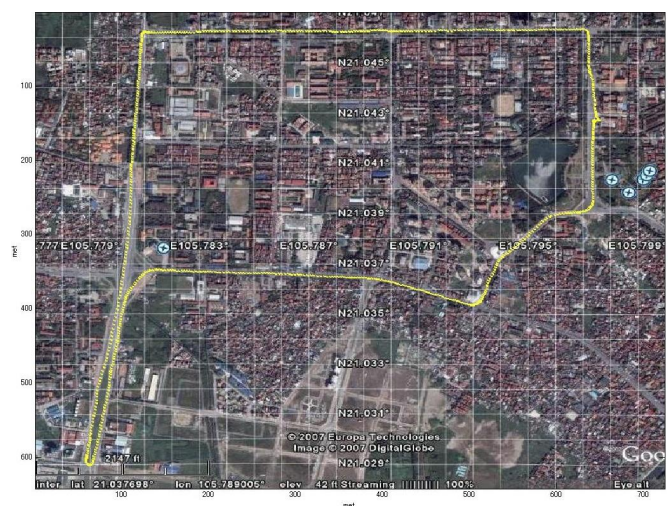

Figure 13. Trajectory of the experimental vehicle.

For the experiment of the IMU on the road [3], GPS and the data acquisition system were installed in a vehicle. The vehicle was driven for 12 minutes in a $5 \mathrm{~km}$ trajectory. Initially the vehicle was at rest, with the engine on, for about 60 seconds in order to estimate the deterministic errors.

The update from the INS was taken every $0.015625 \mathrm{~s}$, the GPS update was taken every $1 \mathrm{~s}$ and the KF was run every $0.5 \mathrm{~s}$ to achieve better accuracy. Figure 12 is the photo of the hardware components of the proposed system. The field test 2-D trajectory is presented in Figure 13.

When the GPS signal is available, the system output can track the GPS trajectory well. In the case where the GPS signal may be lost, the INS can continue to compute the position during the period of lost GPS signal (from the 650th to the 750th seconds). Figure 14(a) presents the trajectory using the feedback configuration of the KF compared with the values measured with the GPS unit. It can be seen that the outputs of the KF (black line) cannot follow the GPS trajectory with a small error for a period of about 100 seconds. The feed-forward KF configuration cannot give a better trajectory error as the feedback one. Otherwise, the combination of the two configurations can give the best results as shown in Figure 14(b). In this combined structure, the feedback KF is used when the GPS signal is available and the feed-forward KF is applied during GPS outages. These figures show the position of the vehicle along the north and east directions on the

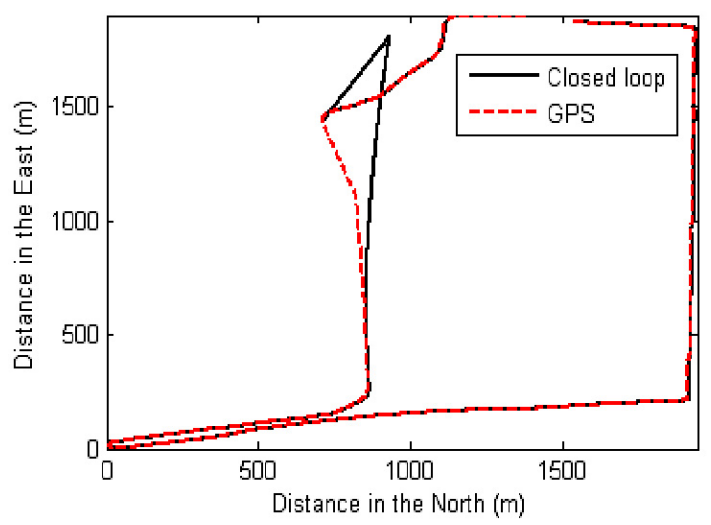

(a)

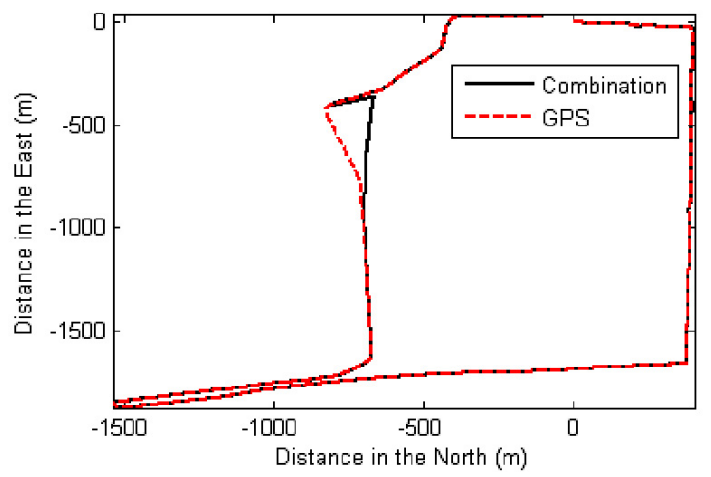

(b)

Figure 14. Comparison of the feedback configuration (a) and the combined one (b) in the case of GPS outage.

Earth instead of latitude and longitude. The reason is to prevent numerical instabilities while calculating the Kalman gain.

Figure 15 shows the roll and pitch of the navigation system. These results seem to be quite reasonable for a system mounted on a land vehicle.

\section{Conclusions}

In this paper, a novel parallel Kalman filter structure was proposed in order to enhance the quality of a combined GPS and INS system. The whole INS/GPS integration system is modeled and simulated using SIMULINK/MATLAB, which is a necessary step towards implementation in real-time hardware. After having observed its operability and its good performance by simulation, this model is embedded into a real system. The experimental results have shown that the initial calibration and alignment are accurate enough to allow navigation with IMU sensors for an extended period of time with low dead reckoning errors. In fact, the parallel Kalman filters structure tremendously improves the accuracy compared to GPS and INS when operating alone as individual navigation systems. The next step of this work is to extend the error models of the INS system. Based on this model, algorithms for in-flight calibration and alignment will be developed and tested for more complex real situations. 

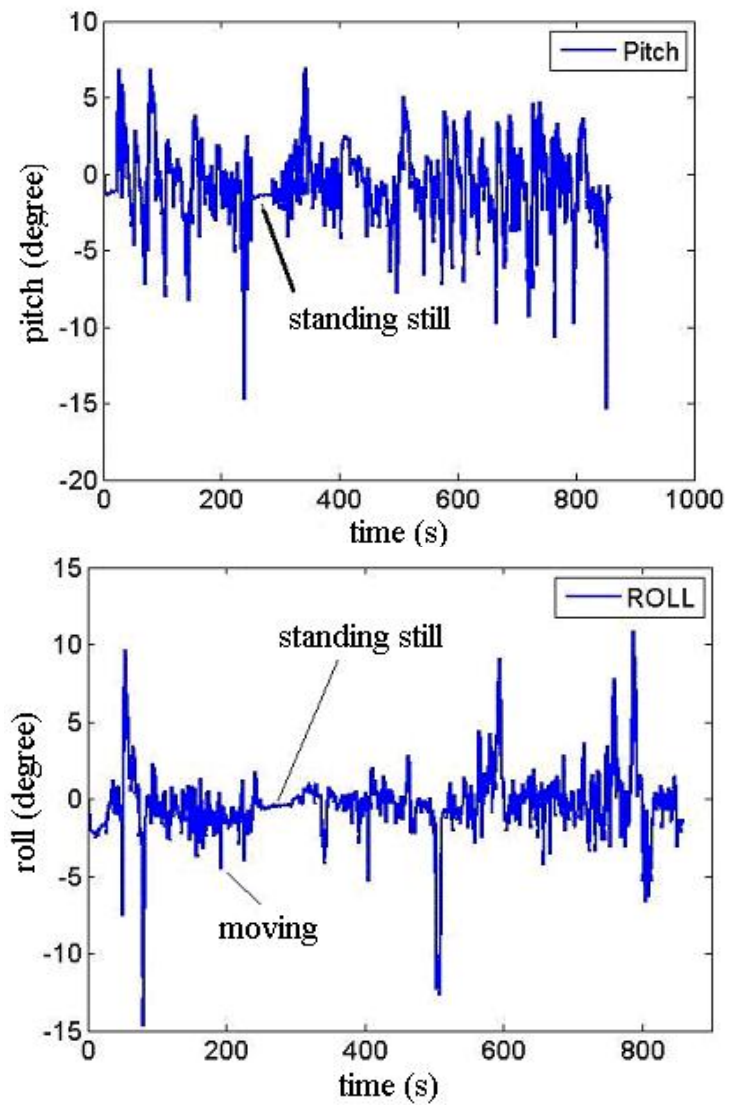

Figure 15. Roll (a) and pitch (b) angles of the integrated navigation system.

\section{ACKNOWLEDGMENTS}

This research was supported by Vietnam National University, Hanoi, under Grant QG.11.31. The authors would like to thank the late Prof. Nguyen Phu Thuy, Dr. Nguyen Van Chuc, Dr. Nguyen Thang Long, and Mr. Luu Manh Ha for contributions in practical implementations.

\section{REFERENCES}

[1] M. Omerbashich, "Integrated INS/GPS navigation from a popular perspective," Journal of Air Transportation, vol. 7, pp. 103-119, 2002.

[2] S. J. Randle and H. M. A, "Low cost navigation using micro-machined technology," in Proc. IEEE Intelligent Transportation Systems Conference, 1997, pp. 1064-1067.

[3] S. Panzieriy, F. Pascucciz, and G. Uliviy, "An outdoor navigation system using GPS and inertial platform," IEEE ASME Transactions on Mechatronics, vol. 7, pp. 134142, 2002.

[4] A. Gelb, Ed., Applied Optimal Estimation. The Massachusetts Institute of Technology Press, 1974.

[5] D. T. Knight, "Rapid development of tightly-coupled GPS/INS system," IEEE Aerospace and Electronics Systems Magazine, vol. 52, pp. 14-18, 1997.

[6] C. Jekeli, Ed., Inertial Navigation Systems with Geodetic Applications. Walter de Gruyter, New York, 2001.

[7] R. Wolf, B. Eissfeller, and G. W. Hein, "A Kalman filter for the integration of a low cost INS and an attitude GPS," in Proc. Int. Symp. Kinematic Systems in Geodesy, Geomatics and Navigation, 26-29 Apr. 1997, pp. 143-150.
[8] E.-H. Shin and N. El-Sheimy, "Accuracy improvement of low cost INS/GPS for land applications," in Proc. 2002 National Technical Meeting of The Institute of Navigation, San Diego, CA, USA, 2002, pp. 146-157.

[9] O. Mezensev, Y. Lu, G. Lachapelle, and R. Klukas, "Vehicular navigation in urban canyons using a high sensitivity GPS receiver augmented with low cost gyro," in Proc. GPS conference, Portland, OR, USA, 24-27 Sept. 2002, pp. 363-369.

[10] E.-H. Shin and N. El-Sheimy, "Accuracy improvement of low cost INS/GPS for land applications," in Proc. 2002 National Technical Meeting of The Institute of Navigation, San Diego, CA, USA, Jan. 28 - 30 2002, pp. 146-157.

[11] A. H. Mohamed and K. P. Schwarz, "Adaptive Kalman filtering for INS/GPS," Journal of Geodesy, pp. 193-203, 1999.

[12] H. Fournier, J. Skaloud, and A. Wägli, "The use of wavelet transform for an automated initialization in GPS/MEMS-IMU integration," in Proc. 13th IAIN World Congress, 2009, pp. 27-30.

[13] V. T. Dau, T. X. Dinh, D. V. Dao, O. Tomonori, and S. Susumu, "Design and fabrication of a convective 3DOF angular rate sensor," in Proc. 6th IEEE Sensors, Atlanta, GA, USA, 28-31 Oct. 2007, pp. 915-918.

[14] T. Mineta, S. Kobayashi, Y. Watanabe, S. Kanauchi, I. Nagakawa, E. Suganuma, and M. Esashi, "Three-axis capacitive accelerometer with uniform axial sensitivities," in Proc. 8th Int. Conf. Solid-State Sensors and Actuators, and Eurosensors IX, Stockholm, Sweden, 25-29 June 1995, pp. 544-577.

[15] R. P. G. Collinson, Ed., Introduction to Avionics. Chapman and Hall, London, 1996.

[16] University of Wisconsin-Madison. Global positioning system (GPS). [Online]. Available: http://www.sco.wisc.edu/gps/system.php

[17] M. S. Grewal, L. R. Weill, and A. P. Andrews, Eds. Kalman Filtering: Theory and Practice using MATLAB. John Wiley and Sons, New York, 2001.

[18] U. BEC Navigation Systems, West Yorkshire. MICRO - ISE BP3010. [Online]. Available: http://www.becnav.co.uk/

[19] O. S. Salychev, Ed., Applied Inertial Navigation: Problems and Solutions. BMSTU Press, 2004.

[20] F. Wang and V. Balakrishnan, "Robust estimators for systems with deterministic and stochastic uncertainties," in Proc. 38th IEEE Conference on Decision and Control, Phoenix, AZ , USA, 07 - 10 Dec. 1999, pp. 1946-1951.

[21] T. D. Tan, L. M. Ha, and N. T. Anh, "A real-time vibration monitoring for vehicle based on 3-DOF MEMS accelerometer," in Proc. 2010 Int. Conf. Computational Intelligence and Vehicular System, 2010, pp. 160-164. 


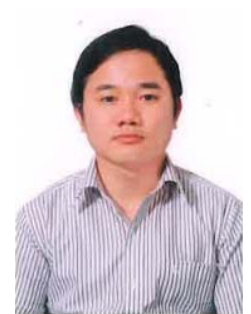

Tran Duc Tan was born in 1980. He received his B.Sc., M.Sc., and Ph.D. degrees respectively in 2002, 2005, and 2010 at the University of Engineering and Technology (UET), Vietnam National University Hanoi, Vietnam (VNUH), where he has been a lecturer since 2006. He is author and coauthor of several papers on MEMS based sensors and their application. His present research interest is in DSP applications.

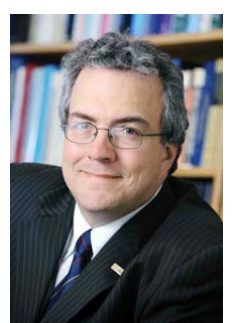

Paul Fortier received his B.Sc. degree and his M.Sc. degree in Electrical Engineering from Université Laval in 1982 and 1984, respectively and his M.S. degree in Statistics and his Ph.D. degree in Electrical Engineering from Stanford University in 1987 and 1989, respectively. Since 1989, he has been with the Department of Electrical and Computer Engineering at Universit $\tilde{A} l^{\prime}$ Laval where he is currently a full professor.

From 1991 to 1996, he was program director for the B.Sc. degree in Computer Engineering and from 1997 to 2003 he was Chairman of the Department of Electrical and Computer Engineering. From 2003 to 2007, he was Associate Dean for Development and Research at the Faculty of Science and Engineering. From 2007 to 2009, he was Vice-president Scientific Affairs and Partnerships at Fonds québécois de la recherche sur la nature et les technologies (FQRNT). Mr. Paul Fortier became Vice-President for Research and Innovation at Université Laval in July 2010.

His research interests include digital signal processing for communications and the study of complexity and performance tradeoffs in hardware implementations, with applications in wireless and optical communications. He has been involved in the organization of national and international conferences and workshops in these fields. He has done consulting work for several companies and government agencies in Canada.

Dr. Fortier is a Fellow of the Engineering Institute of Canada and a Senior Member of the Institute of Electrical and Electronics Engineers (IEEE).

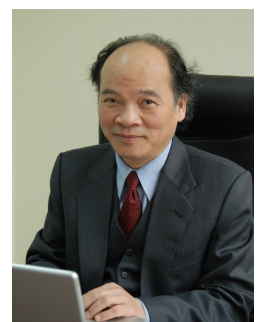

Huu-Tue Huynh was born in Hue, Viet Nam. He received the Sc.D. degree in 1972 from Laval University, Canada, where from 1969 to 2004 he was a faculty member of the Department of Electrical and Computer Engineering. In 2004, he left Laval University to become Chairman of the Department of Data Processing at The College of Technology of the Vietnam National University, Hanoi. Since 2007 he has been President of Bac Ha International University, Vietnam. He was an Invited Guest at The AT\&T Information Systems in Neptune, N.J. in 1984 and has been invited to give lectures at several Universities in Europe, America as well as in Asia. Professor Huynh is author and coauthor of two books and more than two hundred papers in Information Processing. He has served as Consultant to a number of Canadian Government Agencies and Industries. His research interests cover stochastic simulation techniques, information processing, fast algorithms and architectures with applications to finance and to communications. 